\title{
Article
}

\section{Visualization of polynomiography from new higher order iterative methods}

\author{
Saba Freed ${ }^{1}$, Amir Naseem ${ }^{2, *}$ and Muhammad Irfan Saleem ${ }^{3}$ \\ 1 Barani Institute of Sciences, Sahiwal, Pakistan.; sabafreed@gmail.com \\ 2 Department of Mathematics, University of Management and Technology, Lahore 54000, Pakistan. \\ 3 Department of Mathematics, Lahore Leads University, Lahore 54000, Pakistan.; m.irfan_saleem@live.com \\ * Correspondence: amir14514573@yahoo.com
}

Received: 24 August 2019; Accepted: 20 December 2019; Published: 31 December 2019.

\begin{abstract}
Polynomiography is the art and science of visualization in approximation of zeros of polynomials. In this report, we visualize polynomiography of some complex polynomials via iterative methods presented in [1].
\end{abstract}

Keywords: Complex polynomial, root, polynomiography.

MSC: 65H05, 65D32.

\section{Introduction}

$\mathbf{P}$ olynomials are one of the most significant objects in many fields of mathematics. Polynomial root-finding has played a key role in the history of mathematics [2]. It is one of the oldest and most deeply studied mathematical problems. The last interesting contribution to the polynomials root finding history was made by Kalantari [2], who introduced the polynomiography as a method which generates nice looking graphics [3,4]. Polynomiography is defined to be the art and science of visualization in approximation of the zeros of complex polynomials, via fractal and non fractal images created using the mathematical convergence properties of iteration functions [5]. An individual image is called a " polynomiograph "and polynomiography combines both art and science aspects [6,7].

Polynomiography gives a new way to solve the ancient problem by using new algorithms and computer technology [8]. Polynomiography is based on the use of one or an infinite number of iteration methods formulated for the purpose of approximation of the root of polynomials e.g. Newton's method, Halley's method etc. The "polynomiographer " can control the shape and designed in a more predictable way by using different iteration methods to the infinite variety of complex polynomials [9].

According to Fundamental Theorem of Algebra, any complex polynomial with complex coefficients $\left\{a_{n}, a_{n-1}, \ldots, a_{1}, a_{0}\right\}$ :

$$
p(z)=a_{n} z^{n}+a_{n-1} z^{n-1}+\ldots+a_{1} z+a_{0}
$$

or by its zeros (roots) $\left\{r_{1}, r_{2}, \ldots, r_{n-1}, r_{n}\right\}$ :

$$
p(z)=\left(z-r_{1}\right)\left(z-r_{2}\right) \ldots\left(z-r_{n}\right)
$$

of degree $n$ has $n$ roots (zeros) which may or may not be distinct. The degree of polynomial describes the number of basins of attraction and placing roots on the complex plane manually localization of basins can be controlled.

Usually, polynomiographs are colored based on the number of iterations needed to obtain the approximation of some polynomial root with a given accuracy and a chosen iteration method. The description of polynomiography, its theoretical background and artistic applications are described in [2-4]. In this paper, we aim to present polynomiography via iterative methods presented in [1]. 


\section{Iteration}

There are many iterative methods in literture for solving nonlinear equation $f(x)=0$, see for example [10-15]. Here we use following iterative methods [1]:

Algorithm 1. Let $p(z)$ be the complex polynomial, then

$$
\begin{aligned}
y_{n} & =z_{n}-\frac{p\left(z_{n}\right)}{p^{\prime}\left(z_{n}\right)}, n=0,1,2, \ldots, \\
w_{n} & =y_{n}-\frac{p\left(y_{n}\right)}{p^{\prime}\left(y_{n}\right)} \\
z_{n+1} & =w_{n}-\frac{f\left(w_{n}\right)}{f^{\prime}\left(w_{n}\right)}-\frac{f^{2}\left(w_{n}\right) f^{\prime \prime}\left(w_{n}\right)}{2 f^{\prime 3}\left(w_{n}\right)}-\frac{f^{3}\left(w_{n}\right) f^{\prime \prime \prime}\left(w_{n}\right)}{6 f^{\prime 4}\left(w_{n}\right)},
\end{aligned}
$$

which is algorithm (1) for solving nonlinear complex equations.

Algorithm 2. Let $p(z)$ be the complex polynomial, then

$$
\begin{aligned}
y_{n} & =z_{n}-\frac{p\left(z_{n}\right)}{p^{\prime}\left(z_{n}\right)}, n=0,1,2, \ldots, \\
w_{n} & =y_{n}-\frac{p\left(y_{n}\right)}{p^{\prime}\left(y_{n}\right)} \\
z_{n+1} & =w_{n}-\frac{f\left(w_{n}\right)}{f^{\prime}\left(w_{n}\right)}-\frac{f^{2}\left(w_{n}\right) f^{\prime \prime}\left(w_{n}\right)}{2 f^{\prime 3}\left(w_{n}\right)}+\frac{f^{3}\left(w_{n}\right) f^{\prime}\left(y_{n}\right)\left[f^{\prime \prime}\left(w_{n}\right)-f^{\prime \prime}\left(y_{n}\right)\right]}{6 f\left(y_{n}\right) f^{\prime 4}\left(w_{n}\right)},
\end{aligned}
$$

which is algorithm (2) for solving nonlinear complex equations.

Algorithm 3. Let $p(z)$ be the complex polynomial, then

$$
\begin{aligned}
y_{n} & =z_{n}-\frac{p\left(z_{n}\right)}{p^{\prime}\left(z_{n}\right)}, n=0,1,2, \ldots, \\
w_{n} & =y_{n}-\frac{p\left(y_{n}\right)}{p^{\prime}\left(y_{n}\right)} \\
z_{n+1} & =w_{n}-\frac{f\left(w_{n}\right)}{f^{\prime}\left(w_{n}\right)}-\frac{f^{\prime}\left(y_{n}\right) f^{2}\left(w_{n}\right)}{2 f^{\prime 3}\left(w_{n}\right)}\left[\frac{f^{\prime}\left(y_{n}\right)-f^{\prime}\left(w_{n}\right)}{f\left(y_{n}\right)}\left(1-\frac{f^{\prime}\left(y_{n}\right) f\left(w_{n}\right)}{3 f\left(y_{n}\right) f^{\prime}\left(w_{n}\right)}\right)+\frac{f^{\prime}\left(z_{n}\right) f\left(w_{n}\right)\left(f^{\prime}\left(z_{n}\right)-f^{\prime}\left(y_{n}\right)\right)}{3 f\left(z_{n}\right) f\left(y_{n}\right) f^{\prime}\left(w_{n}\right)}\right],
\end{aligned}
$$

which is algorithm (3) for solving nonlinear complex equations.

In above algorithms, $z_{0} \in \mathbb{C}$ is a starting point. The sequence $\left\{z_{n}\right\}_{n=0}^{\infty}$ is called the orbit of the point $z_{o}$ converges to a root $z^{*}$ of $p$ then, we say that $z_{0}$ is attracted to $z^{*}$. A set of all such starting points for which $\left\{z_{n}\right\}_{n=0}^{\infty}$ converges to root $z^{*}$ is called the basin of attraction of $z^{*}$.

\section{Convergence test}

In the numerical algorithms that are based on iterative processes we need a stop criterion for the process, that is, a test that tells us that the process has converged or it is very near to the solution [6-9]. This type of test is called a convergence test. Usually, in the iterative process that use a feedback, like the root finding methods, the standard convergence test has the following form:

$$
\left|z_{n+1}-z_{n}\right|<\varepsilon,
$$

where $z_{n+1}$ and $z_{n}$ are two successive points in the iteration process and $\varepsilon>0$ is a given accuracy. In this paper we also use the stop criterion (2). 


\section{Polynomiographs}

In this section we present some examples of polynomiographs for different complex polynomials equation $p(z)=0$ and some special polynomials using algorithms proposed in [1]. The different colors of a images depend upon number of iterations to reach a root with given accuracy $\varepsilon=0.001$. One can obtain infinitely many nice looking polynomiographs by changing parameter $k$, where $k$ is the upper bound of the number of iterations.

Example 1. The polynomiographs for $z^{2}-1=0$ by using Algorithms 1,2 and 3 are presented here in Figures 1,2 and 3 respectively.

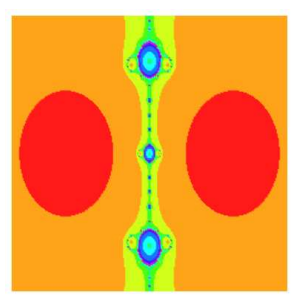

Figure 1. Polynomiograph of $z^{2}-1=0$ via Algorithm 1

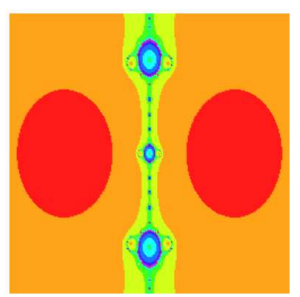

Figure 2. Polynomiograph of $z^{2}-1=0$ via Algorithm 2

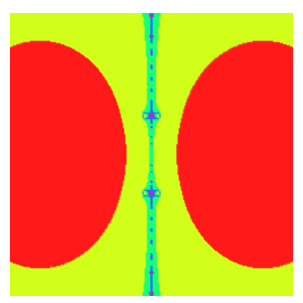

Figure 3. Polynomiograph of $z^{2}-1=0$ via Algorithm 3

Example 2. The polynomiographs for $z^{2}-z+1=0$ by using Algorithms 1, 2 and 3 are presented here in Figures 4,5 and 6 respectively.

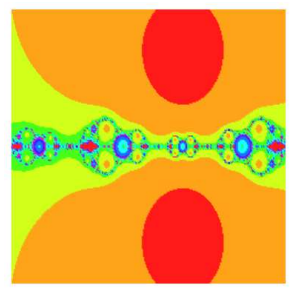

Figure 4. Polynomiograph of $z^{2}-z+1=0$ via Algorithm 1 


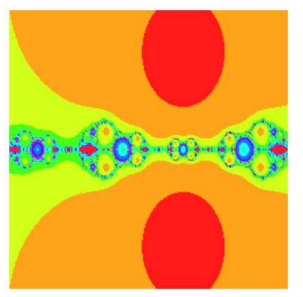

Figure 5. Polynomiograph of $z^{2}-z+1=0$ via Algorithm 2

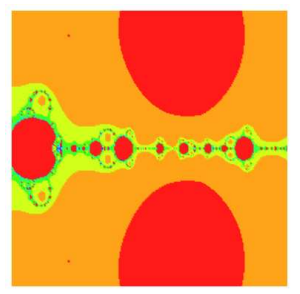

Figure 6. Polynomiograph of $z^{2}-z+1=0$ via Algorithm 3

Example 3. The polynomiographs for $z^{3}-1=0$ by using Algorithms 1,2 and 3 are presented here in Figures 7,8 and 9 respectively.

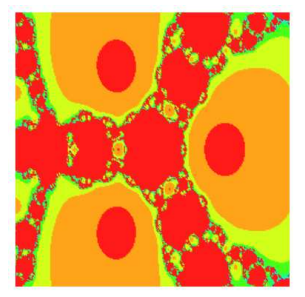

Figure 7. Polynomiograph of $z^{3}-1=0$ via Algorithm 1

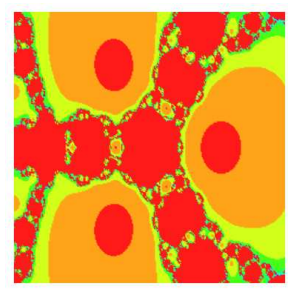

Figure 8. Polynomiograph of $z^{3}-1=0$ via Algorithm 2

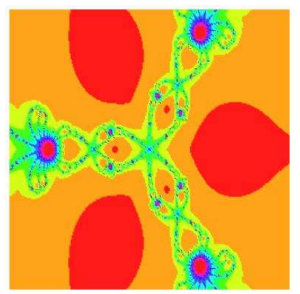

Figure 9. Polynomiograph of $z^{3}-1=0$ via Algorithm 3

Example 4. The polynomiographs for $z^{3}-z-1=0$ by using Algorithms 1, 2 and 3 are presented here in Figures 10, 11 and 12 respectively. 


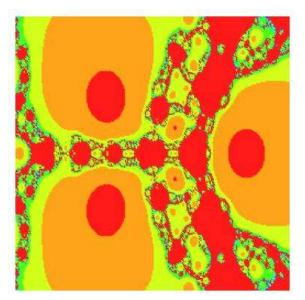

Figure 10. Polynomiograph of $z^{3}-z-1=0$ via Algorithm 1

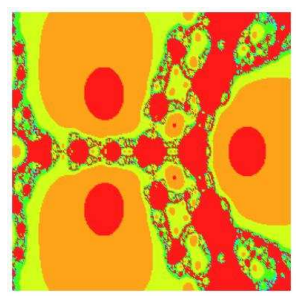

Figure 11. Polynomiograph of $z^{3}-z-1=0$ via Algorithm 2

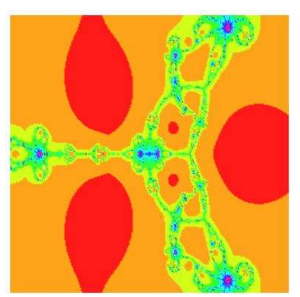

Figure 12. Polynomiograph of $z^{3}-z-1=0$ via Algorithm 3

Example 5. The polynomiographs for $z^{4}-1=0$ by using Algorithms 1, 2 and 3 are presented here in Figures 13,14 and 15 respectively.

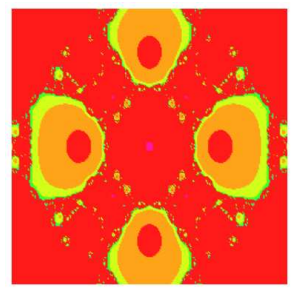

Figure 13. Polynomiograph of $z^{4}-1=0$ via Algorithm 1

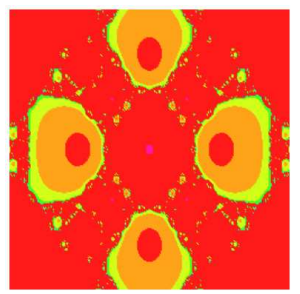

Figure 14. Polynomiograph of $z^{4}-1=0$ via Algorithm 2 


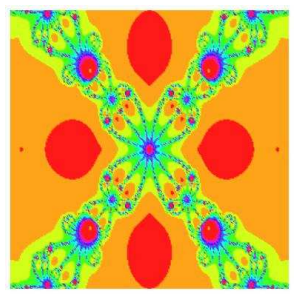

Figure 15. Polynomiograph of $z^{4}-1=0$ via Algorithm 3

Example 6. The polynomiographs for $z^{4}+2 z+1=0$ by using Algorithms 1, 2 and 3 are presented here in Figures 16,17 and 18 respectively.

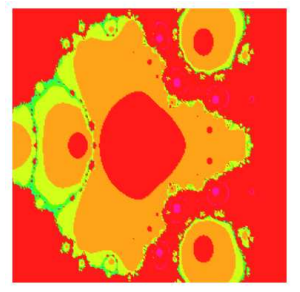

Figure 16. Polynomiograph of $z^{4}+2 z+1=0$ via Algorithm 1

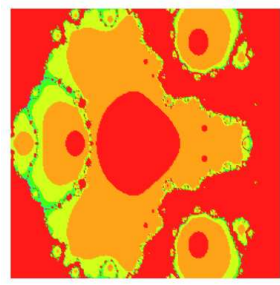

Figure 17. Polynomiograph of $z^{4}+2 z+1=0$ via Algorithm 2

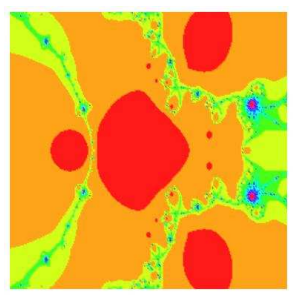

Figure 18. Polynomiograph of $z^{4}+2 z+1=0$ via Algorithm 3

Example 7. The polynomiographs for $z^{5}-1=0$ by using Algorithms 1,2 and 3 are presented here in Figures 19,20 and 21 respectively.

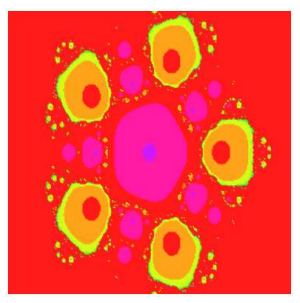

Figure 19. Polynomiograph of $z^{5}-1=0$ via Algorithm 1 


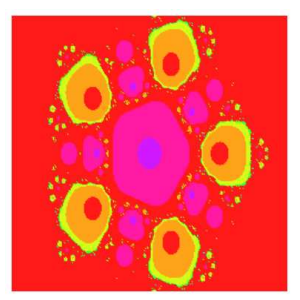

Figure 20. Polynomiograph of $z^{5}-1=0$ via Algorithm 2

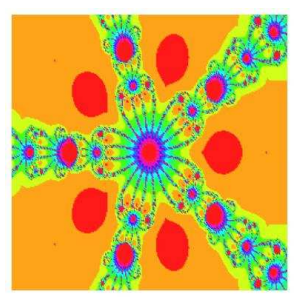

Figure 21. Polynomiograph of $z^{5}-1=0$ via Algorithm 3

Example 8. The polynomiographs for $z^{5}+5=0$ by using Algorithms 1, 2 and 3 are presented here in Figures 22,23 and 24 respectively.

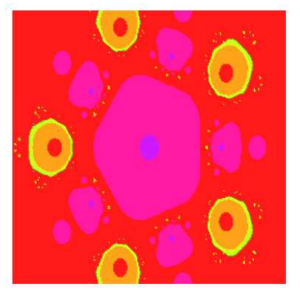

Figure 22. Polynomiograph of $z^{5}+5=0$ via Algorithm 1

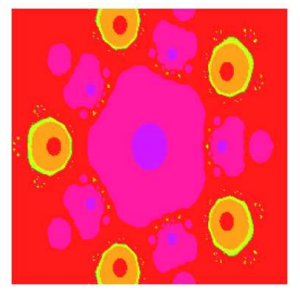

Figure 23. Polynomiograph of $z^{5}+5=0$ via Algorithm 2

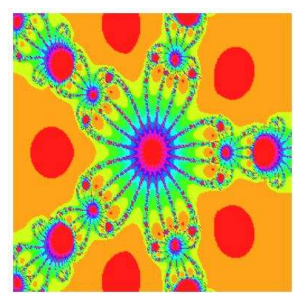

Figure 24. Polynomiograph of $z^{5}+5=0$ via Algorithm 3

Example 9. The polynomiographs for $z^{6}-1=0$ by using Algorithms 1, 2 and 3 are presented here in Figures 25,26 and 27 respectively. 


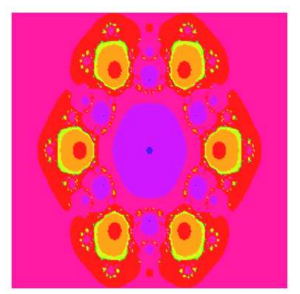

Figure 25. Polynomiograph of $z^{6}-1=0$ via Algorithm 1

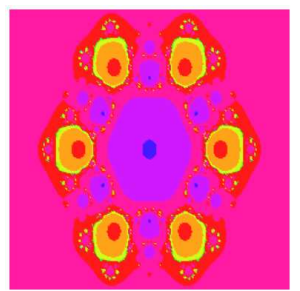

Figure 26. Polynomiograph of $z^{6}-1=0$ via Algorithm 2

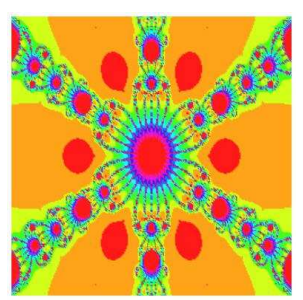

Figure 27. Polynomiograph of $z^{6}-1=0$ via Algorithm 3

Example 10. The polynomiographs for $z^{6}-z^{4}+4=0$ by using Algorithms 1,2 and 3 are presented here in Figures 28, 29 and 30 respectively.

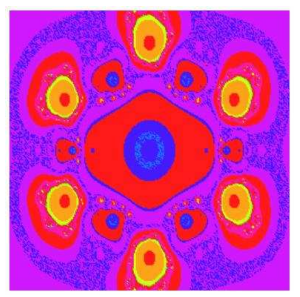

Figure 28. Polynomiograph of $z^{6}-z^{4}+4=0$ via Algorithm 1

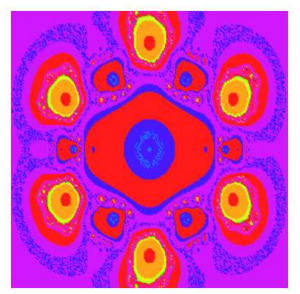

Figure 29. Polynomiograph of $z^{6}-z^{4}+4=0$ via Algorithm 2 


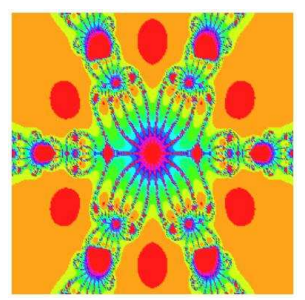

Figure 30. Polynomiograph of $z^{6}-z^{4}+4=0$ via Algorithm 3

Example 11. The polynomiographs for $z^{7}-1=0$ by using Algorithms 1,2 and 3 are presented here in Figures 31,32 and 33 respectively.

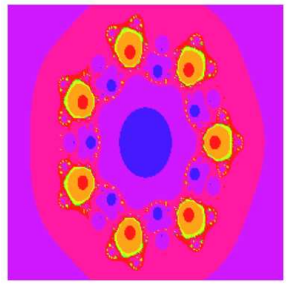

Figure 31. Polynomiograph of $z^{7}-1=0$ via Algorithm 1

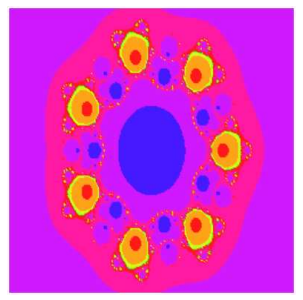

Figure 32. Polynomiograph of $z^{7}-1=0$ via Algorithm 2

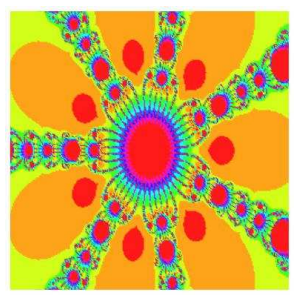

Figure 33. Polynomiograph of $z^{7}-1=0$ via Algorithm 3

Example 12. The polynomiographs for $z^{7}-z^{2}+1=0$ by using Algorithms 1,2 and 3 are presented here in Figures 34,35 and 36 respectively.

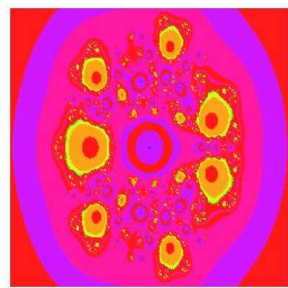

Figure 34. Polynomiograph of $z^{7}-z^{2}+1=0$ via Algorithm 1 


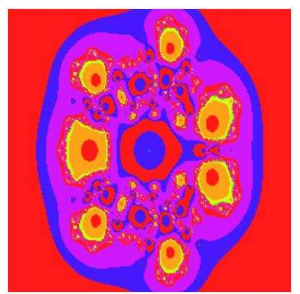

Figure 35. Polynomiograph of $z^{7}-z^{2}+1=0$ via Algorithm 2

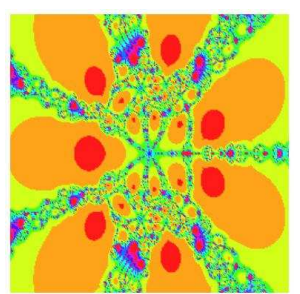

Figure 36. Polynomiograph of $z^{7}-z^{2}+1=0$ via Algorithm 3

Example 13. The polynomiographs for $z^{8}-1=0$ by using Algorithms 1,2 and 3 are presented here in Figures 37,38 and 39 respectively.

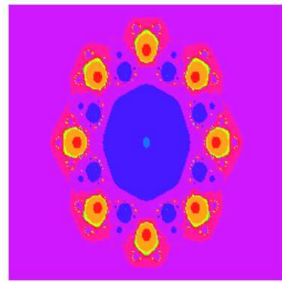

Figure 37. Polynomiograph of $z^{8}-1=0$ via Algorithm 1

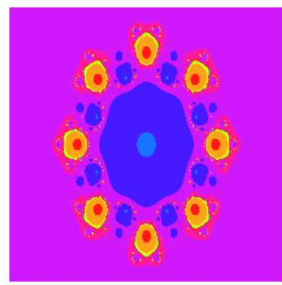

Figure 38. Polynomiograph of $z^{8}-1=0$ via Algorithm 2

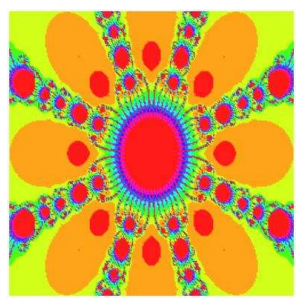

Figure 39. Polynomiograph of $z^{8}-1=0$ via Algorithm 3

Example 14. The polynomiographs for $z^{8}+8=0$ by using Algorithms 1,2 and 3 are presented here in Figures 40,41 and 42 respectively. 


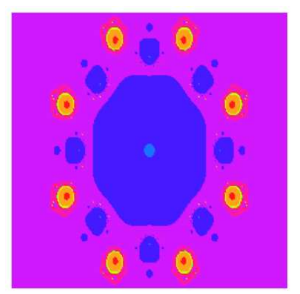

Figure 40. Polynomiograph of $z^{8}+8=0$ via Algorithm 1

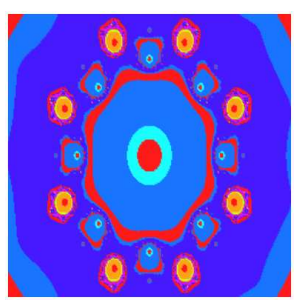

Figure 41. Polynomiograph of $z^{8}+8=0$ via Algorithm 2

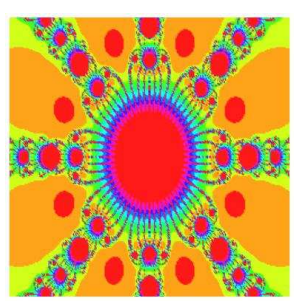

Figure 42. Polynomiograph of $z^{8}+8=0$ via Algorithm 3

Example 15. The polynomiographs for $z^{9}-1=0$ by using Algorithms 1,2 and 3 are presented here in Figures 43,44 and 45 respectively.

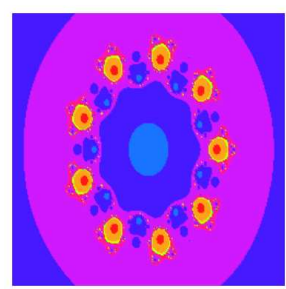

Figure 43. Polynomiograph of $z^{9}-1=0$ via Algorithm 1

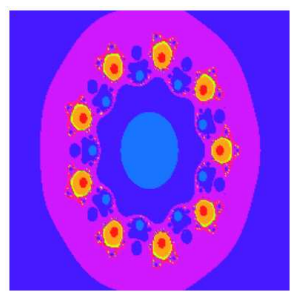

Figure 44. Polynomiograph of $z^{9}-1=0$ via Algorithm 2 


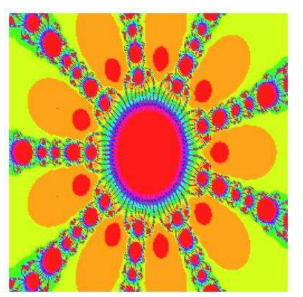

Figure 45. Polynomiograph of $z^{9}-1=0$ via Algorithm 3

Example 16. The polynomiographs for $z^{10}-1=0$ by using Algorithms 1,2 and 3 are presented here in Figures 46,47 and 48 respectively.

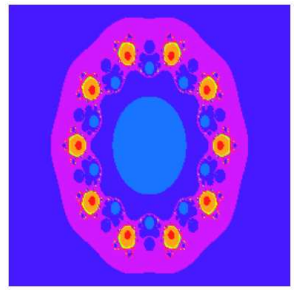

Figure 46. Polynomiograph of $z^{10}-1=0$ via Algorithm 1

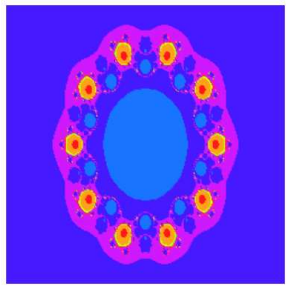

Figure 47. Polynomiograph of $z^{10}-1=0$ via Algorithm 2

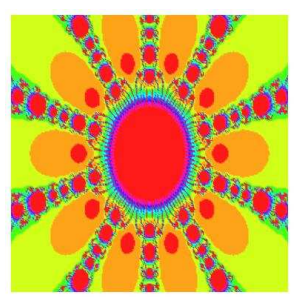

Figure 48. Polynomiograph of $z^{10}-1=0$ via Algorithm 3

Author Contributions: All authors contributed equally to the writing of this paper. All authors read and approved the final manuscript.

Conflicts of Interest: "The authors declare no conflict of interest."

\section{References}

[1] Saba, S., Naseem, A., \& Saleem, M. I. (2019). Modified Abbasbandy's method free from second derivative for solving nonlinear equations. Open Journal of Mathematical Sciences, 3(1), 109-114.

[2] Kalantari, B. (2005). Method of creating graphical works based on polynomials. U.S. Patent No. 6,894,705. Washington, DC: U.S. Patent and Trademark Office.

[3] Kalantari, B. (2005). Polynomiography: from the fundamental theorem of Algebra to art. Leonardo, 38(3), $233-238$.

[4] Kotarski, W., Gdawiec, K., \& Lisowska, A. (2012, July). Polynomiography via Ishikawa and Mann iterations. In International Symposium on Visual Computing (pp. 305-313). Springer, Berlin, Heidelberg. 
[5] Bahman, K. (2008). Polynomial root-finding and polynomiography. World Scientific. Publishing Co., New Jersey.

[6] Naseem, A., Nazeer, W., \& Awan, M. W. (2016). Polynomiography via modified Abbasbanday's method. Science International(Lahore), 28(2), 761-766.

[7] Ali, A., Naseem, A., Munir, M., \& Nizami, A. R. (2016). Polynomiography via generalized Newton-Rapshon method free from second derivative. Science International(Lahore), 28(3), 2425-2430.

[8] Naseem, A., Munir, M., Nizami, A. R., \& Athar, M. (2016). Dynamics of a new three step iterative method. Science International(Lahore), 28(3), 2431-2438.

[9] Naseem, A., Munir, M., Nizami, A. R., \& Athar, M. (2016). Polynomiography via Traub's method. Science International(Lahore), 28(3), 2431-2438.

[10] Nazeer, W., Naseem, A., Kang, S. M., \& Kwun, Y. C. (2016). Generalized Newton Raphson's method free from second derivative. Journal of Nonlinear Sciences and Applications, 9 (2016), 2823, 2831.

[11] Noor, M. A. (2007). Some iterative methods free from second derivatives for nonlinear equations. Applied Mathematics and Computation, 192(1), 101-106.

[12] Noor, M. A., \& Khan, W. A. (2012). Fourth-Order Iterative Method Free from Second. Applied Mathematical Sciences, 6(93), 4617-4625.

[13] Nazeer, W., Kang, S. M., \& Tanveer, M. (2015). Modified Abbasbandy's Method for Solving Nonlinear Functions with Convergence of Order Six. International Journal of Mathematical Analysis, 9(41), 2011-2019.

[14] Nazeer, W., Tanveer, M., Rehman, K., \& Kang, S. M. (2016). Modified new sixth-order fixed point iterative methods for solving nonlinear functional equations. International Journal of Pure and Applied Mathematics, 109, $223-232$.

[15] Chun, C., \& Kim, Y. I. (2010). Several new third-order iterative methods for solving nonlinear equations. Acta applicandae mathematicae, 109(3), 1053-1063. 